\title{
ANÁLISIS DEL SISTEMA DE SALUD DEL ECUADOR
}

\author{
ANALYSIS OF ECUADORIAN HEALTH SYSTEM
}

\author{
ANALISE DO SISTEMA DE SAÚDE DO EQUADOR
}

\section{RICARDO CAÑIZARES FUENTES', GABRIELA MENA RIBADENEIRA', GIAFFAR BARQUET ABI-HANNA'}

\author{
${ }^{1}$ Universidad Católica de Santiago de Guayaquil, Guayaquil, Ecuador
}

Introducción: Ecuador inició una reforma en su sistema de salud en el año 2008; sin embargo, los cambios generados por la nueva Constitución de la República, en la salud pública y el acceso a atención de salud en el Ecuador, han sido escasamente evaluados y documentados. Objetivo: examinar la experiencia del Ecuador en la reforma de su sistema de salud analizando algunas de las áreas que han sufrido cambios como parte de este proceso. Metodología: se realizó investigación de tipo descriptivo, observacional. Se recuperó artículos relevantes de bases de datos que incluyó PubMed, Cochrane, ScienceDirect y UpToDate, se consultó sitios web gubernamentales y de organizaciones relacionadas con la salud como el Banco Mundial, Organización Mundial de la Salud, Organización Panamericana de la Salud, Unión de Naciones Suramericanas, entre otras. Resultados: se destaca en este periodo la decisión política de centralizar la planificación y gestión de salud a nivel del gobierno central. Se incrementa el número de consultas de morbilidad pero sin incremento de las de prevención. Las enfermedades crónicas ocupan los primeros lugares como causa de muerte. La mortalidad materna se mantiene alta y no alcanza la meta propuesta por el país. Hay importante incremento del presupuesto en salud, pero este no refleja protección financiera de los pacientes ya que el gasto de bolsillo está cerca del 50\%. Conclusión: el sistema de salud del Ecuador no logra aún alcanzar plenamente objetivos sanitarios que impacten en la mejora de la calidad de la atención de la salud. También persisten barreras de acceso a atención integral y oportuna; y, limitada protección financiera. Se requiere consolidar sistemas de información que permitan acceso a información completa y oportuna.

PALABRAS CLAVE: Sistema de Salud, políticas públicas de salud, salud pública, sistemas de información en salud.

Abstract

Introduction: Ecuador initiated a reform in its health system in 2008; however, changes in public health and access to health care in Ecuador generated by the new Constitution have been poorly evaluated and documented. Objective: To examine reform of Ecuadorian health system by analyzing some of the areas that have undergone changes as part of this process. Design: Observational descriptive research. Relevant articles were retrieved from databases that included PubMed, Cochra-ne, ScienceDirect and UpToDate; government websites and organizations related to health such as the World Bank, World Health Organization, Pan American Health Organization, Union of South American Nations, among others were consulted. Results: The political decision to centralize health planning and management at the central government level was highlighted during this period. Morbidity is increased but there is no increase in prevention. Chronic diseases occupy first places as a cause of death. Maternal mortality remains high and does not reach the goal proposed by the country. There is significant increase in the health budget, but this does not reflect the financial protection of the patients since the out-of-pocket expense is close to $50 \%$. Conclusion: The health system of Ecuador does not yet achieve full health objectives that impact on the improvement of health quality and health care. There are also barriers to access comprehensive and timely care and limited financial protection. It is necessary to consolidate information systems that allow access to complete and timely information.

KEYwORDS: health systems, public health policy, public health, health information systems.

Resumo

Introdução: Equador iniciou uma reforma em seu sistema de saúde em 2008, porém, as mudanças geradas pela nova constituição na saúde publicam e 0 aceso a atenção de saúde no Equador tem tido escassamente avaliados e documentados. Objetivo: examinar a expertise do equador na reforma do seu sistema de saúde, analisando algumas das áreas que tem sofrido mudanças como parte deste processo. Metodology: se realizo investigação de tipo descritivo observacional. Foram recuperados artículos importantes da base de dados que incluiu PubMed, Cochra-ne, ScienceDirect y UpToDate, se consultou paginas web governamentais e de organizações relacionadas com a saúde como o banco mundial, organização mundial da saúde, organização panamericana da saúde, união das nações sul-americanas, entre outras. Resultados: foi destacado neste período a decisão politica de centralizar 0 planejamento e gestão em saúde ao nível do governo central. Se incrementa o numero de consultas de morbilidade mas sem incremento das consultas de prevenção. As doenças crônicas são a primeira causa de morte. A mortalidade materna continua alta e não alcança a meta do pais. Tem importante incremento do orçamento em saúde, mas não da proteção financeira aos pacientes porque eles gastam cerca de $50 \%$. Conclusão: 0 sistema de saúde do equador não consegue ainda alcançar plenamente objetivos sanitários que melhorem a qualidade atenção da saúde. Também continuam barreiras de acesso a atenção integral e oportuna e limitada proteção financeira. É preciso consolidar sistemas de informação que permitam o acesso a informação completa e oportuna

Palabras-chave: Sistemas de Saúde, políticas públicas de saúde, saúde pública, sistemas de informação em saúde. 
INTRODUCCIÓN

La investigación en Sistemas de Salud (SS) ha ido rápidamente aumentando a nivel mundial y con métodos más rigurosos para evaluarlo, ha ganado espacio en la agenda académica y política. ${ }^{1}$ De acuerdo a la Organización Mundial de la Salud (OMS), un sistema de salud consiste de todas las organizaciones, personas y acciones cuyo primer objetivo es el de promover, restaurar o mantener la salud. Este concepto incluye además todos los esfuerzos necesarios para influir en los determinantes de la salud. ${ }^{2}$

Las investigaciones en SS se han basado en comprender los elementos clave de éstos, examinando los roles desempeñados por los actores y/o involucrados en los mismos y en promover estrategias para mejorar su performance y su respuesta ante las emergentes necesidades de salud de la población. ${ }^{1}$

Hoy en día, también se centran en cómo estos SS se deberían adaptar a los avances científicos y en la adopción de nuevas tecnologías. ${ }^{1}$ Pero existe poca información sobre la capacidad para enfrentar los nuevos retos de la salud mundial, como son las enfermedades no transmisibles, sus altos costos y las brechas existentes en proteger a toda la población.

Resulta valioso examinar un sistema de salud desde el punto de vista no sólo de sus funciones y sus ámbitos de acción, sino también en relación a las reformas que éste ha sufrido y a las limitaciones y/u obstáculos por los cuales pudiera atravesar. ${ }^{1}$ Mientras existe literatura global sobre SS en diferentes regiones del mundo, especialmente en Europa donde se cuenta con un observatorio de SS, ${ }^{3}$ en el Ecuador la información sobre el SS y cómo este resuelve las necesidades de salud de los ecuatorianos, es limitada y poco accesible.

Ecuador inició una importante reforma en su sistema de salud en el 2008, para entenderlo, es necesario partir desde el marco legal y normativo sobre el cual se basa esta reforma sanitaria. ${ }^{4}$

En el año 2008, el Ecuador tuvo una reforma en la Constitución de la República, la cual rige hasta el día de hoy. En este nuevo marco legal, se reconoce que el Estado garantiza el derecho a la salud, que se vincula con el ejercicio de otros derechos como acceso al agua, alimentación, nutrición, educación, cultura física, trabajo, seguridad social, ambientes sanos y otros que sustenten el buen vivir. ${ }^{4-6}$ El cumplimiento de estos derechos es responsabilidad del Estado a través de políticas económicas, sociales, culturales, educativas y ambientales, así como el acceso permanente a programas, acciones y servicios de promoción y atención integral de salud, salud sexual y reproductiva. ${ }^{4-7}$ Los cambios generados por la nueva Constitución en la salud pública y el acceso a atención de salud en el Ecuador han sido escasamente evaluados y documentados. Por esta razón, el objetivo de este artículo es examinar la experiencia del Ecuador en la reforma de su sistema de salud analizando algunas de las áreas que han sufrido cambios como partes de este proceso. ${ }^{7}$ El análisis del sistema de salud se realizó usando enfoques de la Unión de Naciones Suramericanas (UNASUR) y de la Organización Panamericana de Salud (OPS), adaptados a nuestra realidad nacional. ${ }^{8}$

Este artículo tiene la finalidad de analizar la evolución del sistema de salud del Ecuador entre el 2010 y 2016 como parte de un estudio en el que se está relacionando el funcionamiento del sistema de salud y los efectos de los desastres naturales, específicamente los terremotos (Proyecto efectos de terremoto en Pedernales en el funcionamiento de Sistema de Salud. Estudio de caso Manabí-Chone).

Se espera proveer evidencia que ayude a los directivos, gestores, investigadores, políticos y sociedad civil del Ecuador a realizar un balance entre los avances del sistema de salud y sus retos pendientes, contribuyendo con insumos para establecer acuerdos políticos y técnicos en un proceso con alta participación ciudadana ya que estamos convencidos que es el mecanismo más adecuado para mejorar sistemática y progresivamente los SS de los ecuatorianos.

\section{METODOLOGÍA}

Se realizó investigación de tipo descriptivo, documental. Dado que la literatura revisada tenía una amplia gama de metodologías empleadas, y teniendo en cuenta el alcance y la naturaleza del objetivo, se eligió un enfoque narrativo para este manuscrito. Una versión modificada de Green et al. (2006).

\section{FUENTES DE INFORMACIÓN}

Se realizaron tres búsquedas separadas de la literatura para identificar información relevante sobre el SS del Ecuador, se insertaron las palabras clave y los términos relacionados para realizar la búsqueda que cubrió al análisis del SS e indicadores de atención en salud. 
La búsqueda original comenzó a inicios del 2017 y continuó hasta octubre del mismo año. Después de recuperar artículos relevantes de la biblioteca de la UCSG, se realizaron búsquedas en bases de datos individuales para ampliar su alcance, así como a través de las bases de datos PubMed, Cochrane, ScienceDirect y UpToDate, desde el 2012 hasta el 2017.

La búsqueda se realizó utilizando las siguientes palabras clave y términos MeSH y/o DeCS: "health systems", "sistemas de salud", "Ecuador”. Los operadores booleanos como 'OR', 'AND' y 'NOT' se usaron para combinaciones de palabras clave y términos MeSH. Se buscaron estos términos en los campos de título, resumen y palabras clave de los artículos.

Se buscó literatura gris a través de una búsqueda en Google para encontrar información sobre el SS del Ecuador y sus indicadores entre los sitios web gubernamentales y de organizaciones relacionadas con la salud, así como publicaciones de organismos internacionales como el Banco Mundial, Organización Mundial de la Salud, Organización Panamericana de la Salud, entre otras.

Otras fuentes de información incluyen a las nacionales como el Instituto Nacional de Estadísticas y Censos (INEC) y el MSP. Como resultado de estas consultas, se recabó información adicional, como conferencias y datos publicados. La disponibilidad y tipo de los datos sobre ciertos indicadores fue muy variable, lo que limita la comparabilidad entre los artículos, los términos de búsqueda y delimitación.

CRITERIOS DE SELECCIÓN

Los criterios de inclusión para la elegibilidad de los artículos se limitaron a los escritos en idioma español e inglés, trabajos de investigación, meta-análisis, revisiones sistemáticas e informes gubernamentales y de organizaciones nacionales e internacionales vinculadas a la salud que se centraron en el desempeño de los SS, a nivel internacional y del Ecuador. Esta búsqueda recuperó 28 documentos. Los artículos que se incluyeron en el estudio fueron 16, que se agruparon luego en grupos temáticos para evaluarlos.

RESULTADOS

Se identificaron los siguientes aspectos con énfasis en el periodo 2010-2016: principales cambios del SS del Ecuador, el comportamiento de los problemas de salud que afectan a los ecuatorianos en el periodo analizado, y debilidades y avances en el funcionamiento del SS.

Para facilidad de interpretación, se agrupó los resultados por ejes temáticos:

\section{RECTORÍA}

En relación a la rectoría, que es la capacidad de conducción del sistema de salud a nivel nacional, la Constitución del 2008 enfatiza que es el Ministerio de Salud Pública (MSP) el organismo encargado de regular el sector salud. ${ }^{4}$ Así mismo, el MSP es quien debe encargarse del control y vigilancia del cumplimiento de las normas establecidas en la Constitución. ${ }^{4}$

En esta función se destaca en este periodo la decisión política de centralizar la planificación y gestión de salud a nivel del gobierno central, y una desconcentración de ciertas funciones técnico administrativas a los niveles zonales y locales.

El país fue dividido en zonas y distritos de salud que son circunscripciones geográfico-poblacionales, que empiezan a funcionar desde el 2012. Las unidades de salud (US) básicas se clasifican dependiendo de su nivel de complejidad en A, B y C. Las US tipo A, cuentan sólo con personal médico y de enfermería; las de tipo B cuenta con atención odontológica, obstétrica, medicina general y pediatría; mientras que las de tipo $C$, ofrecen a su vez maternidad e internamiento. ${ }^{12}$ Cada distrito elabora y ejecuta el presupuesto de su Plan Operativo Anual (POA), el presupuesto es enviado a la Dirección de la Zona y ésta lo remite al MSP en Quito, la capital del Ecuador, donde se toman las decisiones finales.

El MSP tiene un gran crecimiento en dependencias técnicas reflejada en la conformación de dos viceministerios. Se crea la red pública y complementaria de salud y el tarifario de prestaciones médicas; ${ }^{5}$ se emite normativas y regulaciones y se incrementa la infraestructura reflejada en nuevos hospitales y centros de salud implementados y ubicados conforme lo indica el Ministerio de salud en base a una metodología que considera cobertura poblacional y capacidad de resolución, ${ }^{5}$ se inicia la implementación del modelo de atención de salud familiar y comunitario y se dan cambios en la concepción de la participación social . 
Esta reestructuración del Ministerio de salud que, acorde a la reestructuración de todas las funciones del Estado, pasó de una estructura con niveles nacional, provincial y de áreas de salud a una estructura conformada por niveles nacional, zonal y distrital. Este cambio no ha sido evaluado aún para medir su beneficio en relación al modelo anterior.

También se crearon nuevas dependencias como la Agencia de Regulación y el Control de la Calidad, Seguridad, Eficacia e Inocuidad de los productos de uso y consumo humano (ARCSA) en el 2012, ${ }^{10}$; y la Agencia de Aseguramiento de la Calidad de los Servicios de Salud (ACSES) en el 2015, como instancia especializada, para promover y controlar la calidad de servicios públicos y privados de salud, adscrita al Ministerio de salud. ${ }^{11}$ También en el 2009 se crea la empresa pública de fármacos, ENFARMA EP, con Decreto Ejecutivo 181, con el propósito de proveer medicamentos de calidad, a precios adecuados, a la red pública integral de salud; sin embargo, por diferentes problemas, desaparece pocos años después.

Fueron eliminados organismos como el Servicio de Erradicación de la Malaria (SNEM) que cumplía un rol importante en el control de enfermedades transmitidas por vectores; otras instituciones se reorganizaron como el Instituto Nacional de Higiene "Leopoldo Izquieta Pérez" que pasó a ser el Instituto Nacional de Investigaciones en Salud Pública (INSPI), derivando algunas funciones a la ARCSA.

El MSP también ejerce la rectoría de las entidades prestadoras de servicios de salud a través de la regulación de las mismas por medio del otorgamiento de un permiso de funcionamiento que rige tanto para empresas de salud pública o privadas y/o de medicina prepagada.

Adicionalmente la Contraloría General de Estado se encarga de controlar los hospitales y unidades médicas en el ámbito del gasto público. Esta entidad examina y evalúa la gestión financiera, administrativa y operativa da las entidades donde el Estado tiene participación. ${ }^{4}$

PROVISIÓN DE SERVICIOS

El número de establecimientos del Ministerio de salud se incrementa en el periodo estudiado, especialmente de hospitales básicos que pasan de 84 a 89 en el 2106, los hospitales generales pasan de 26 a 32 de unidades de salud. (Tabla 1)
TABLA 1. NÚMERO DE ESTABLECIMIENTOS DEL MSP POR TIPOS, 2010-2016. HOSPITALES BÁSICOS Y GENERALES.

\begin{tabular}{lcr}
\hline AÑO & HOSPITALES BÁSICOS & HOSPITALES GENERALES \\
\hline 2010 & 84 & 26 \\
\hline 2011 & 84 & 26 \\
\hline 2012 & 84 & 26 \\
\hline 2013 & 85 & 29 \\
\hline 2014 & 87 & 25 \\
\hline 2015 & 84 & 29 \\
\hline 2016 & 89 & 32 \\
\hline
\end{tabular}

Fuente: INEC. Recursos y actividades de salud 2010-2016.

Respecto a unidades de salud del primer nivel de atención (subcentros y centros de salud) no hay mayores cambios, inclusive disminuye el número total de estas unidades de salud de 1470 a 1437 en el 2016; sin embargo es evidente el incremento de centros de salud tipo C que tienen mayor capacidad de resolución. (Tabla 2).

\section{TABLA 2. UNIDADES DE PRIMER NIVEL}

\begin{tabular}{rrrrr}
\hline AÑO & CENTROSA & CENTROS B & CENTROSC & TOTAL \\
\hline 2010 & 1470 & & & 1470 \\
\hline 2011 & 1507 & & & 1507 \\
\hline 2012 & 1521 & & & 1521 \\
\hline 2013 & 1540 & 21 & 20 & 1581 \\
\hline 2014 & 1534 & 18 & 17 & 1569 \\
\hline 2015 & 1367 & 8 & 68 & 1443 \\
\hline 2016 & 1362 & 10 & 65 & 1437 \\
\hline
\end{tabular}

Fuente: INEC. Recursos y actividades de salud 2010-2016.

Si se considera el total de establecimientos de atención del MSP, hubo un crecimiento regular de los mismos hasta el año 2013 en que se estabiliza, para decaer el 2016; y si se compara con el crecimiento poblacional, el porcentaje de incremento de unidades del 2010 al 2016 representó un $58 \%$ con respecto al crecimiento poblacional. (Figura 1).

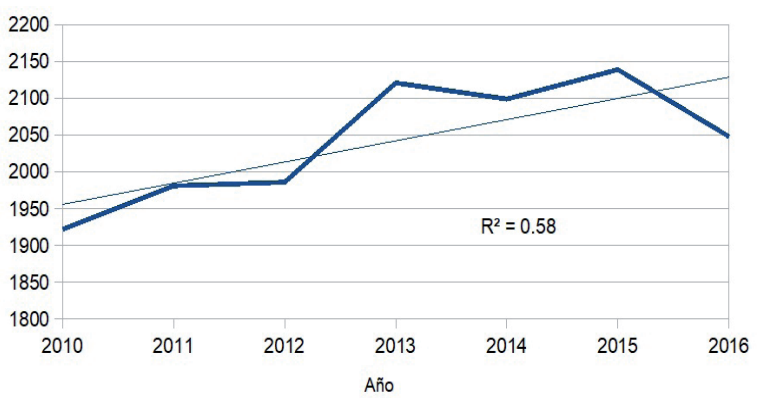

Figura 1. Establecimientos de salud, MSP 2010-2016. Fuente: INEC. Elaborado: Autores.

Respecto a las visitas domiciliarias, si bien se evidencia crecimiento, cabe recalcar que hasta 
el año 2007 el INEC registró de manera desagregada las visitas domiciliarias por consultas de prevención ejecutadas por médicos, obstetrices y enfermeras de las visitas domiciliarias por consultas de morbilidad, distribuidas así mismo según el profesional que las ejecutó. A partir de ese año hasta el último reporte publicado (2016), se han agrupado todas estas actividades en una sola variable.

La comparación entre el crecimiento poblacional y las visitas domiciliarias ejecutadas por el MSP, muestra desde el 2001 una tendencia continua de crecimiento de estas últimas, a la par con la población $(\mathrm{r}=0,723)$ aunque esa respuesta representa solo un $53 \%$ de la demanda estimada. (Figura 2).

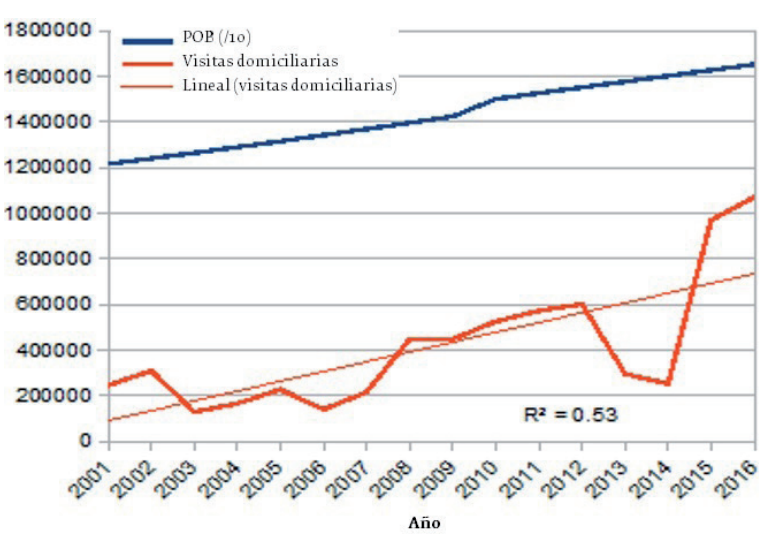

Figura 2. Visitas domiciliarias 2001-2016.

Fuente: INEC. Elaborado: Autores.

Llama la atención la importante caída de esta actividad durante los años 2013 y 2014 para luego presentar un incremento importante durante los 2 años siguientes.

Respecto a las consultas ambulatorias, es evidente que crece el número de consultas, pero falta información para saber si éstas se traducen en resolutivas del problema de salud que se consulta o si el problema se resuelve en la red de SS del distrito o zona de salud y no tener que viajar a las grandes ciudades que finalmente significa mayores costos al paciente y su familia.

En relación a camas disponibles, el MSP la define así: "camas habilitadas en las salas del establecimiento, en condiciones de uso inmediato, para la atención de los pacientes hospitalizados, independiente de que estén ocupadas o no". No se deben considerar las camas deterioradas o en mantenimiento ni las cunas. (Figura 3).

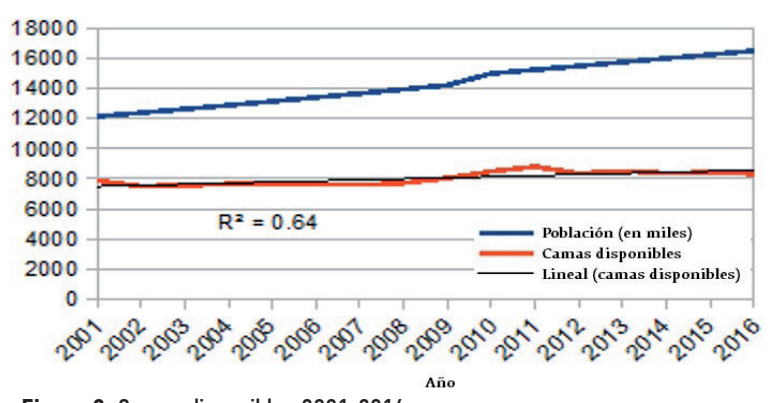

Figura 3. Camas disponibles 2001-2016.

Fuente: INEC. Elaborado: Autores.

El número de camas disponibles en los establecimientos del MSP ha tenido un incremento sostenido desde 2001, pero no se incrementa conforme a las necesidades de la población ya que el mismo representa el $64 \%$ con respecto al crecimiento de la población.

Esta situación se reafirma al analizar el número de camas reportadas por el INEC, las camas disponibles a nivel nacional, el sistema de salud ecuatoriano contaba con 23.784 camas en el 2010, 24.545 en el 2011, 23.138 en el 2012, 23.667 en el 2013 y a 24.634 en el 2014. ${ }^{13}$ Este último dato arroja una razón de 1.5 camas por 1000 habitantes, la cual está por debajo de la recomendación de la OPS de 2,4 camas por $1000 \mathrm{~h}$.

COMPORTAMIENTO DE PROBLEMAS DE SALUD 2010-2016 Para el análisis del comportamiento de problemas de salud se consideraron datos de las gacetas epidemiológicas del MSP, así como datos de morbilidad del Anuario de Estadísticas Hospitalarias: (egresos y camas 2014 del INEC). En relación a las principales causas de morbilidad a nivel nacional, se evidencia que las enfermedades infecciosas continúan apareciendo en los primeros lugares. (Tabla 3).

\section{TABLA 3. DIEZ PRINCIPALES CAUSAS DE MORBILIDAD, 2014.}

\begin{tabular}{|c|c|c|c|}
\hline $1^{\circ}$ & K80 & Colelitiasis & $36,147 \quad 3.03 \% \quad 22.55$ \\
\hline $2^{\circ}$ & K35 & Apendicitis aguda & $35,566 \quad 2.98 \% \quad 22.19$ \\
\hline $3^{\circ}$ & A09 & $\begin{array}{l}\text { Diarrea y gastroenteritis de presunto } \\
\text { origen infeccioso }\end{array}$ & $30,269 \quad 2.54 \% \quad 18.89$ \\
\hline $4^{\circ}$ & $\mathrm{J} 18$ & Neumonía, organismo no especificado & $29,994 \quad 2.51 \% \quad 18.71$ \\
\hline $5^{\circ}$ & N39 & Otros trastornos del sistema urinario & $17,183 \quad 1.44 \% \quad 10.72$ \\
\hline $6^{\circ}$ & 006 & Aborto no especificado & $15,783 \quad 1.32 \%$ \\
\hline $7^{\circ}$ & $\mathrm{K} 40$ & Hernia inguinal & $15,201 \quad 1.27 \%$ \\
\hline $8^{\circ}$ & 023 & $\begin{array}{l}\text { Infección de las vías genitourinarias en } \\
\text { el embarazo }\end{array}$ & $12,951 \quad 1.09 \%$ \\
\hline $9^{\circ}$ & 047 & Falso trabajo de parto & $12,585 \quad 1.06 \%$ \\
\hline $10^{\circ}$ & 034 & $\begin{array}{l}\text { Atención materna por anormalidades } \\
\text { conocidas o presuntas de los órganos } \\
\text { pelvianos de la madre }\end{array}$ & $12,241 \quad 1.03 \%$ \\
\hline
\end{tabular}


Sin embargo, al revisar las principales causas de muerte, seevidencia quelas enfermedades crónicas no transmisibles (ENT) ocupan los primeros lugares en el 2014, (Tabla 4) convirtiéndose en un importante reto a resolver ya que por un lado son prevenibles, pero a la vez, son causas de muertes prematuras ${ }^{14}$ La gripe y neumonía son las únicas enfermedades transmisibles (ET) que aparecen dentro del cuadro, lo cual refleja una transición epidemiológica de las ET a las ENT. El análisis se enfocó en enfermedades trazadoras como las trasmitidas por agua y alimentos, reportes de brotes epidémicos, mortalidad materna y enfermedades vectoriales que reflejarían condiciones sanitarias y además el impacto de los programas de control. Se construyeron, para cada una de estas entidades, tasas sobre 100.000 habitantes, de acuerdo a las proyecciones de población para el país, proporcionadas por el INEC; por limitaciones de información se analizó período 2014-2016 y parte del 2017.

\section{TABLA 4. PRINCIPALES CAUSAS DE MUERTE, 2014.}

\begin{tabular}{lllrr}
\hline Código $^{\text {a }}$ & Causas de muerte & №. & $\%$ & Tasa \\
\hline I20-I25 & Enfermedad isquémica del corazón & 4430 & 7,0 & 27,64 \\
\hline E10-E14 & Diabetes mellitus & 4401 & 6,9 & 27,46 \\
\hline I60-169 & Enfermedades cerebrovasculares & 3777 & 6,0 & 23,57 \\
\hline I10-115 & Enfermedades hipertensivas & 3572 & 5,6 & 22,29 \\
\hline J10-J18 & Gripe yneumonía & 3418 & 5,4 & 21,33 \\
\hline V00-V89 & Accidentes de transporte terrestre & 3059 & 4,8 & 19,09 \\
\hline K70-K76 & Cirrosis y otras enfermedades del hígado & 2038 & 3,2 & 12,72 \\
\hline N00-N39 & Enfermedades del sistema urinario & 1712 & 2,7 & 10,68 \\
\hline J40-J47 & Enfermedades crónicas del sistema respiratorio & 1656 & 2,6 & 10,33 \\
\hline C16 & Neoplasia maligna & 1585 & 2,5 & 9,89 \\
\hline
\end{tabular}

aSegún la clasificación estadística internacional de enfermedades y problemas relacionados con la salud, décima revisión. .Tasa por 100000 habitantes.

Fuente: INEC. Compendio estadístico 2014

La información analizada sobre enfermedades transmitidas por vectores, fue la reportada hasta las primeras semanas de 2016 como paludismo y parcialmente del 2015, para dengue y chikungunya. Llama la atención la tendencia al incremento de los casos de paludismo, el cual, en años anteriores, había reducido su transmisión, así como la presencia de epidemias de dengue y chikungunya en el 2015. (Tabla 5).

\section{TABLA 5. ENFERMEDADES TRANSMITIDAS POR VECTORES.}

\begin{tabular}{lrrr}
\hline ETV/Año & 2014 & 2015 & 2016 \\
\hline Paludismo & 0,54 & 3,43 & $1,39^{*}$ \\
\hline Dengue & 96,38 & $260,97^{* *}$ & \\
\hline Chikungunya & 0,17 & $260,97^{* * *}$ & \\
\hline
\end{tabular}

*Hasta la semana 20. ** Hasta la semana 40. ${ }^{* * *}$ Hasta la semana 40.

ETV: enfermedades transmitidas poro vectores.

Fuente: MSP. Gaceta epidemiológica 2014-2017. INEC. Proyecciones de población 2010-2020.
En relación con los reportes de brotes epidémicos se observa un incremento hacia 2015 y luego descenso en el 2017. (Figura 4).

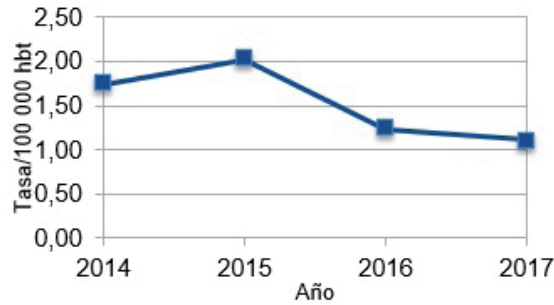

Figura 4. Reporte de brotes epidémicos, 2014-2017.

Fuente: MSP. Gaceta epidemioloógica 2014-2017. INEC, proyecciones de población 2010-2020.

Elaborada: Autores.

Similar comportamiento fue el observado con las enfermedades transmitidas por agua y alimentos. (Figura 5).

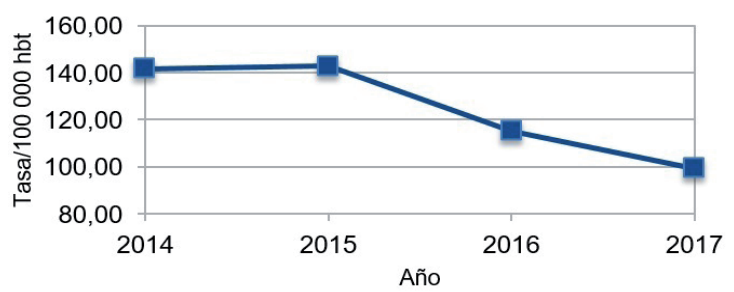

Figura 5. Enfermedades transmitidas por agua y alimentos, 2014-2017. Fuente: MSP. Gaceta epidemioloógica 2014-2017. INEC, proyecciones de población 2010-2020.

Elaborado: Autores.

Diferente es el comportamiento de la mortalidad materna que luego de descender hacia el 2015 mantiene una tendencia de crecimiento hasta 2017, lo que indicaría que el Ecuador no alcanzó la meta de los objetivos del milenio.

Este indicador, es uno de los indicadores que mejor refleja el acceso a medidas de prevención y acceso a atención de salud de calidad en un país. (Figura 6).

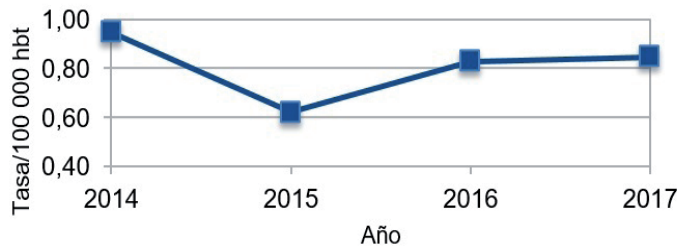

Figura 6. Mortalidad materna, 2014-2017.

Fuente: MSP. Gaceta epidemioloógica 2014-2017. INEC, proyecciones de población 2010-2020.

Elaborado: Autores.

\section{RECURSOS HUMANOS}

Respecto al personal sanitario, progresivamente se ha ido mejorando la densidad de recursos humanos, especialmente de médicos y enfermeras de tal forma que el Ecuador según la OPS 
alcanzó la meta de los desafíos de Toronto que para el 2015 se debía tener 25 profesionales de la salud por 10.000 habitantes ${ }^{14}$. Se pasó de 11201 a 33644 profesionales entre los años 2008 y $2015^{5}$, situación que reflejaría una continuidad de la política de incrementar los recursos humanos en el Ministerio de Salud, en este periodo.

Sin embargo, otras metas de los desafíos de Toronto no se alcanzaron, por ejemplo la razón de enfermeras por médico, la proporción de empleo precario, que debería reducirse a la mitad. Tampoco se alcanzó la meta de que la proporción de médicos de atención primaria para el 2015 debía exceder el $40 \%$ de la fuerza laboral médica total. En estos indicadores el Ecuador está por debajo de lo alcanzado por Chile, Perú y Colombia. ${ }^{15}$

Pero el incremento del personal de salud no logra disminuir las inequidades en la distribución de personal de salud entre zonas urbanas y rurales, conforme lo indica la OPS la tasa urbana de médicos fue de 29,01 y la tasa rural de 5,42 por 10.000 habitantes, ${ }^{14,16}$ lo que implicaría que la mayor cantidad de personal de salud están en las grandes ciudades donde están los principales hospitales y menor personal de salud para la atención primaria.

\section{ACCESO Y DISPONIBILIDAD DE MEDICAMENTOS}

La disponibilidad de medicamentos en el Ecuador es influenciada por su producción, lo cual implica un tema de equidad social en el acceso a los mismos, derecho a la salud y control del poder económico controlado por la industria farmacéutica. ${ }^{17}$ Los productos farmacéuticos son uno de las principales mercados económicos del mundo pero desafortunadamente la falta de estos, continua siendo un problema para los países en vías de desarrollo como Ecuador. ${ }^{17,19}$

En un intento por solucionar este problema, en el año 2000 se aprobó la Ley de medicamentos genéricos, que obliga a las instituciones públicas a emplear fármacos genéricos equivalentes a los patentados. ${ }^{4}$ Esta iniciativa redujo el margen de beneficio de las farmacias con respecto a los productos farmacéuticos patentados y promovió el mayor expendio de los genéricos. ${ }^{4}$

Años después, en diciembre de 2009, se crea la primera empresa pública de fármacos, denominada "ENFARMA EP". ${ }^{18}$ Entre las funciones de esta empresa, a más de la producción de medicamentos, estaban la de investigar y promover el desarrollo de principios activos y la comercialización de medicamentos genéricos y patentados tanto para humanos, animales y plantas. ${ }^{19}$ Sin embargo, en Ecuador en el sector público solamente hay disponibilidad del $42 \%$ de la lista de medicamentos considerados "esenciales" por la OMS y aproximadamente el $71 \%$ está disponible en el sector privado. ${ }^{17}$ Esto posiciona al Ecuador en el puesto 26 de peor accesibilidad en una lista de 38 países en vías de desarrollo. ${ }^{17}$

En el 2016 ENFARMA EP fue liquidada debido a que no logró cumplir sus objetivos. ${ }^{18,19}$

Ecuador predomina el consumo de medicamentos patentados en lugar de usar genéricos. Al comparar al Ecuador con Estados Unidos, se observa que nueve de cada diez medicamentos que se consumen, son patentados (de marca), mientras que en Estados Unidos se utiliza un $90 \%$ de medicamentos genéricos. ${ }^{19}$

Acceso. Aproximadamente la mitad del presupuesto en salud de los hogares ecuatorianos es destinado exclusivamente a medicamentos, siendo las familias más pobres las mayormente afectadas. ${ }^{17}$ En una investigación de Ortiz-Prado et $a{ }^{20}$ que utilizó datos de la Encuesta Nacional de Ingresos y Gastos de los Hogares (ENIGHUR) 2010 del INEC, se demostró que es en las familias más pobres donde se refleja un mayor gasto de bolsillo en medicamentos vs las familias más pudientes. En los primeros deciles de pobreza las personas gastan el $67 \%$ del total de gasto de bolsillo en medicamentos, mientras que en los deciles superiores, este gasto es del $51 \% .^{20}$

La última ENIGHUR, 2011-2012 muestra una pequeña diminución del gasto de bolsillo, del $67 \%$ en el 2010 al 64\% para el periodo 2011-2012; sin embargo, aún se observa una brecha en la cual las familias de más recursos gastan un porcentaje menor que las familias más pobres. ${ }^{17}$ (Figura 7).

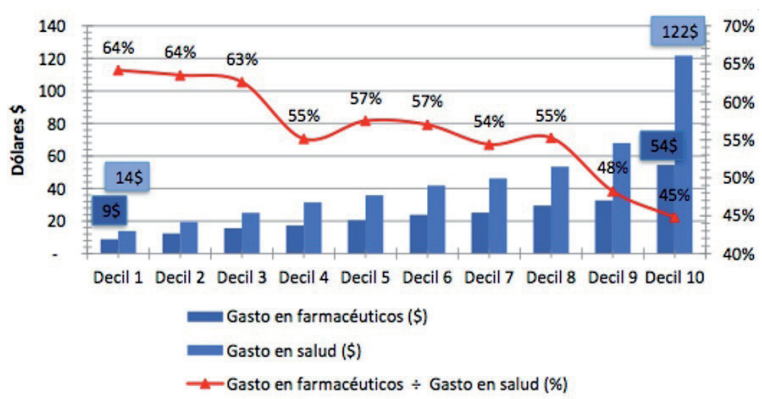

Figura 7. Gasto promedio por hogar en productos farmacéuticos y gasto en salud, 2014-2017. Fuente: La PPEDA, DONUM SF. Enfarma enferma y muere Problemas de la industria farmacéutica pública en el Ecuador.

Elaborado: Autores. 
En conclusión, el acceso a medicamentos es una cuestión de equidad social, bajo los escenarios antes mencionados, se observa que las familias con menos recursos sacrifican más de la mitad de su presupuesto en salud para la adquisición de medicamentos, impidiendo que sea invertido en otros servicios como medicina preventiva o gastos en seguridad social.

FINANCIAMIENTO

La Constitución estipula que el financiamiento público en salud deberá de ser oportuno, regular y suficiente y que el mismo debe provenir del Presupuesto General del Estado. (REF) Actualmente existe también el Seguro General Obligatorio (SGO) del Instituto Ecuatoriano de Seguridad Social (IESS) cuyo fondo de financiamiento proviene de tres fuentes: 1. El aporte individual de los afiliados al mismo, 2. La aportación obligatoria de los empleadores y 3. La contribución del Estado.

Otras instituciones como el Instituto de Seguridad Social de la Policía Nacional (ISSPOL) y el Instituto de Seguridad Social de las Fuerzas Armadas (ISSFA) también ejercen un aporte al financiamiento del sistema de salud del Ecuador. ${ }^{3}$ Las fuentes de financiamiento del sistema nacional de salud además de las provenientes del sector público (IESS, ISSPOL, ISSFA) incluyen además al sector privado el cual es mayormente representado por los seguros privados y planes de medicina prepagada, y que incluyen las primas a algún tipo de seguro privado, primas al seguro de accidentes de tránsito, pagos por complementarios y pagos por servicios prestados varios, entre otros. Otras fuentes comprenden el sector externo en el cual se incluyen los recursos de cooperación. Tabla 6 .

TABLA 6. FUENTES DE FINANCIAMIENTO DEL SS DEL ECUADOR.

SECTOR PÚBLICO SECTOR PRIVADO SECTOR EXTERNO

Impuestos generales Primas a algún tipo de Recursos de

seguroprivado cooperación

\begin{tabular}{ll}
\hline Impuestos específicos & $\begin{array}{l}\text { Copago por servicios } \\
\text { asegurados }\end{array}$ \\
\hline $\begin{array}{l}\text { Cotizaciones a los seguros } \\
\text { sociales públicos }\end{array}$ & $\begin{array}{l}\text { Pago directos por servicio } \\
\text { prestados }\end{array}$ \\
\hline & $\begin{array}{l}\text { Pagos complementarios } \\
\text { (medicamentos, insumos) }\end{array}$ \\
\hline & Primas al seguro de \\
& accidentes de tránsito \\
\hline
\end{tabular}

Fuente: ISAGS. Sistemas de salud en Suramérica: desafíos para la universalidad la integralidad y la equidad. Rio de Janeiro: ISAGS; 2012.

En cuanto al gasto en salud, en el que se incluye el gasto público y el privado ha aumentado constantemente en los últimos 10 años. De 5.9\% del PIB en 2008 pasó al 7.3\% en el 2011. ${ }^{8}$ El gasto per cápita en salud aumentó de 241,97 USD en el 2008 a 579,19 USD en el 2014 según datos del Banco Mundial. ${ }^{21}$ El dato que refleja cercanamente el gasto público en salud es el Presupuesto Sectorial Salud (devengado) (PSS) como \% del PIB se observa una tendencia a la alza hasta el 2013 donde alcanza su acmé para posteriormente descender en el 2014.

Este PSS parte de un 1,4\% en el 2008 y asciende a 2,3\% en el 2013. (Figura 8). Este porcentaje está por debajo de la recomendación de la OPS de que se necesita asignar un $6 \%$ del presupuesto público para que un sistema de salud cubra las atenciones de salud de la población.

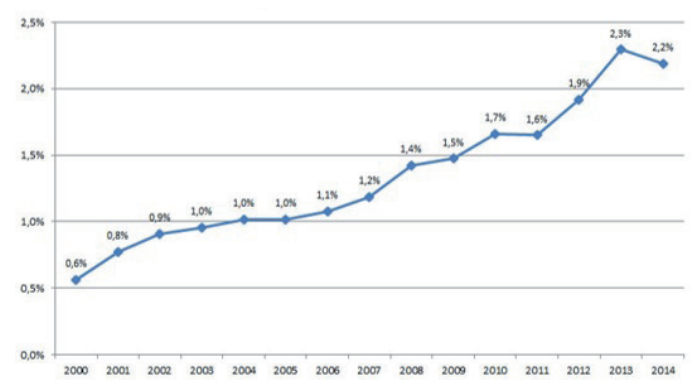

Figura 8. Presupuesto sectorial salud como \% del PIB (devengado). Fuente: Banco Central del Ecuador y Ministerio de Finanzas.

Una baja inversión pública en salud impacta en la economía de la población, así al analizar el gasto de bolsillo, las cifras han aumentado. Según datos publicados por el Banco Mundial, ${ }^{21} \mathrm{el}$ gasto de bolsillo en el Ecuador ha aumentado de un $44,31 \%$ en el 2013 , a un $48,42 \%$ en el 2014 . Este valor es expresado como porcentaje del gasto total en salud (Out-of-pocket health expenditure, \% of total expenditure on health). ${ }^{22}$ Esto nos indica que casi la mitad del costo de la atención en salud proviene del usuario, demostrando así que el gasto privado en salud es aún comparado con países europeos o suramericanos como Colombia.

\section{DISCUSIÓN}

El principal evento que explica los diferentes cambios del sistema de salud se da en el ámbito político con la Constitución aprobada en el 2008 la que define al sistema de salud de acceso universal y gratuito y basado en un modelo de atención familiar y comunitario.

A partir del 2008 las diferentes gestiones técnicopolíticas que han conducido el Ministerio de 
salud han procurado avanzar en la dirección señalada por la Constitución. Sin embargo, no ha sido un avance continuo, ha existido etapas de avances y otras de enlentecimiento incluso de reformulación de enfoques y programas de salud.

Por ejemplo, entre el 2007-2010 se hizo énfasis en la propuesta de Trasformación del Sector Salud, en el periodo 2013-2016 se plantea la reforma del sector salud, ${ }^{23,24}$ lo cual también se refleja en la propuesta del modelo de atención basado en la atención primaria. En un inicio se implementaron los equipos básicos de salud (EBAS), posteriormente los equipos de técnicos de atención de salud (TAPS) y finalmente el programa médico de barrio. Si bien estos programas al parecer proponen lo mismo, será necesario evaluar y evitar que cada gestión política que llega al Ministerio de salud no este reiniciando programas sino se persiga la continuidad en las políticas públicas, con los justes que resulten de las evaluaciones.

Esta poca continuidad o falta de sumatoria de mejoras progresivas, no ha permitido la consolidación del modelo de atención primaria en estos años, lo cual se refleja en la alta demanda de atención de morbilidad en los hospitales y centros de salud y poco impacto de las acciones a nivel domiciliario y comunitario en la prevención de los problemas prevalentes de salud en la comunidad. Se podría decir que en Ecuador el modelo de atención primaria está en proceso de implementación ${ }^{12}$ y necesita fortalecerse y consolidarse para que produzca impacto beneficioso en la población.

Los datos encontrados demuestran que si bien se han dado avances en el periodo analizado, éstos no logran alcanzar las metas sanitarias internacionales como la disponibilidad suficiente de personal de salud ni el número de camas hospitalarias, ni reducción de la mortalidad materna, conforme a los compromisos adquiridos a nivel internacional por el país, lo que ubica al Ecuador por debajo de otros países de la región como Chile, Uruguay y Colombia. ${ }^{23}$ Esta situación implica que no toda la población tenga acceso real a atención de salud.

Si los objetivos de un SS se enfocan en el mejoramiento de la salud de la población, en una adecuada respuesta a las expectativas de las personas y a la protección financiera frente a los riesgos, el impacto debe reflejarse en que toda la población esté accediendo a una atención de salud integral y oportuna, en el mejoramiento de indicadores sanitarios como disminución de la mortalidad materna, en disminución de la presencia de brotes epidémicos y disminución del gasto de bolsillo ${ }^{24}$ en ese sentido el sistema de salud del Ecuador no logra aun alcanzar plenamente esos objetivos. No es suficiente el aumento del número de consultas médicas; éstas deben reflejarse en un impacto real para la población.

En ese sentido la prevención de enfermedades trasmisibles de prioridad en la salud pública como el VIH no desciende en su transmisión y se presentaron brotes de dengue, chikungunya y zika, y la malaria está presentando brotes en Esmeraldas y amazonia. Si un sistema de salud es sólido debe prevenir o controlar adecuada y oportunamente los brotes epidémicos, su presencia es un indicador de que el componente preventivo tiene falencias. Esto puede deberse al énfasis en el sistema curativo.

Situación que también se relaciona con la brecha de recursos humanos en salud especialmente a nivel de atención primaria de salud.

Una acción importante en este periodo es la conformación de la red pública integral de salud, en la que en base a un tarifario nacional del costo de las prestaciones médicas el sector privado complementa con servicios de atención médica que ni el Ministerio de Salud ni el Seguro Social tienen suficiente oferta. Falta consolidar la red pública integral de salud mejorando la calidad, cobertura y oportunidad de la atención así como se recomienda implementar adecuadas medidas de control, el uso del presupuesto así como evitar supuestos actos de corrupción que desvía importantes recursos del presupuesto público. ${ }^{25}$

Si bien la dotación de recursos humanos, especialmente de médicos, se ha incrementado, persiste la inequidad en la distribución del personal de salud y la falta de especialistas ha implicado que en este periodo haya permanente problemas de disponibilidad de citas médicas asignadas oportunamente. La mayoría de especialistas se encuentran concentrados en las grandes ciudades, lo cual se convierte en barreras de acceso para la población rural y de ciudades pequeñas o intermedias.

En cuanto al financiamiento si bien hay una mayor asignación económica presupuestada, esta 
no alcanza los niveles recomendados por la OMS/ OPS del $6 \%$ del PIB y si comparamos con países de la región como Chile, Uruguay Colombia y Brasil, el Ecuador está distante aun de la inversión que hacen estos países. Como consecuencia el gasto de bolsillo de los ciudadanos es alto cerca del 50 \% en comparación con sistemas de salud como el Colombiano o de países europeos pertenecientes a la Organización para la Cooperación y el desarrollo económico (OECD) que es alrededor de $20 \% .{ }^{26} \mathrm{El}$ mayor porcentaje del gasto de bolsillo va para medicamentos, lo cual también refleja debilidades del sistema de salud y constituyen barreras económicas para el tratamiento de las enfermedades.

Respecto a la participación social en el periodo analizado a criterio de los autores, se debilita, ya que desaparecen los consejos cantonales y provinciales de salud, el Conseja Nacional de Salud disminuye su rol asignado en la anterior Constitución y se pone en funcionamiento a partir del 2012 el Consejo Ciudadano sectorial de salud con el objetivo de convertirse en instancia sectorial de diálogo, deliberación y seguimiento de las políticas públicas de carácter nacional y sectorial, ${ }^{27}$ pero sus aportes han sido limitados.

Por lo tanto un reto a trabajar a futuro es fortalecer la participación ciudadana ${ }^{28}$ en la preparación e implementación de las políticas publica. Si bien es necesario fortalecer la Rectoría del Ministerio de salud esta debe ser complementada con la participación de la sociedad civil de tal forma que se mejore la gobernabilidad en salud que implica construir procesos de dialogo y consensos que respalden la sostenibilidad de las políticas públicas.

Una limitante del estudio fue la poca disponibilidad de información que permita tener datos completos tanto sobre los indicadores sanitarios como datos relacionados con la provisión de servicios de salud y su financiamiento, lo cual también es una limitante para la planificación, evaluación y diseño de políticas públicas en salud. Se requiere consolidar sistemas de información o construir observatorios del funcionamiento del sistema de salud. ${ }^{24}$

CONCLUSIONES

En el periodo analizado se evidencia avances como la conformación de la red pública integrada de salud, el incremento del personal de salud, incremento de unidades de salud y de equipamiento médico; sin embargo, no ha sido un avance continuo ni integral en todo el sistema; han existido etapas de enlentecimiento incluso de reformulación de políticas, enfoques y planes de salud.

Esta poca continuidad y el peso del enfoque biomédico que enfatiza la atención hospitalaria y los intereses que giran a su alrededor, no ha permitido la consolidación del modelo de atención primaria en estos años, lo cual se refleja en la alta demanda de atención de morbilidad en los hospitales y centros de salud y el poco impacto de las acciones a nivel domiciliario y comunitario en la prevención de los problemas prevalentes de salud en la comunidad.

En ese sentido el sistema de salud del Ecuador no logra aún alcanzar plenamente objetivos sanitarios que impacten en la mejora de la calidad y atención de salud. No es suficiente el aumento del número de consultas médicas, estas deben reflejarse en un impacto real para la población. Por ejemplo la mortalidad materna no desciende a niveles aceptados internacionalmente y la persistencia de brotes epidémicos y la demora en su control son indicadores de que el componente preventivo es débil.

También persisten barreras de acceso a la atención integral, y oportuna de la salud así como limitada protección financiera que se refleja en que aproximadamente la mitad del presupuesto en salud de los hogares ecuatorianos es destinada exclusivamente a medicamentos, siendo las familias más pobres, las mayormente afectadas.

Se necesita cerrar las brechas de acceso y atención que todavía persisten, así como tener capacidad de resiliencia para superar los efectos de la inestabilidad política y económica que afectan el desempeño del sistema de salud.

La participación ciudadana requiere ser fortalecida sin pretender institucionalizarla como una función más del Estado; eso le quita autonomía, representatividad, capacidad crítica y exigibilidad.

Se requiere crear y consolidar sistemas de información que permitan acceso a las bases de datos completas, continúas y oportunas, lo cual mejorará la participación ciudadana, así como la planificación e implementación de políticas públicas y su evaluación. 
CONSIDERACIONES ÉTICAS

Esta investigación no involucra la recopilación de datos primarios de los participantes; por tanto, se descarta cualquier conflicto de intereses; sin embargo, fue realizado en base a los principios éticos de la declaración de Helsinski de 1964 para las investigaciones médicas en seres humanos.

\section{LIMITACIONES DE LA INVESTIGACIÓN}

La búsqueda de información demostró que existe limitada literatura sobre el SS de Ecuador y la información sobre algunos indicadores sanitarios no está disponible y en unos casos un mismo indicador tiene diferente valor ya que la literatura evaluada reflejó una amplia gama de metodologías.

\section{REFERENCIAS BIBLIOGRÁFICAS}

1. Roncarolo F, Boivin A, Denis J-L, Hebert R, Lehoux P. What do we know about the needs and challenges of health systems? A scoping review of the international literature. BMC Health Serv Res. 8 de septiembre de 2017;17(1):636.

2. World Health Organization. Everybody's businessstrengthening health systems to improve health outcomes: WHO's framework for action. 2007;

3. OECD. Health at a Clance 2017: OECD Indicators [Internet]. 2017. Disponible en: www.oecd.org/ health/health-at-a-glance.htm

4. Lucio R, Villacres N, Henriquez R. The health system of Ecuador. Salud Publica Mex. 2011;53 Suppl 2:s177-187.

5. Espinosa V, Acuña C, de la Torre D, Tambini C. La reforma en salud del Ecuador. 2017; Rev Panam Salud Publica 41, 2017, disponible en: http://iris.paho. org/xmlui/bitstream/handle/123456789/34061/ v41a962017.pdf?sequence $=1$ \&isAllowed $=y$

6. Luna C, Emanuele CA, De La Torre D. Posicionamiento de Ecuador en la agenda de salud global como producto de la reforma sectorial. 2017; Rev Panam Salud Publica 41, 2017 http://iris.paho. org/xmlui/bitstream/handle/123456789/34060/ v41a552017.pdf?sequence $=1$ \&isAllowed $=y \& u a=1$

7. Constitución de la República del Ecuador [Internet]. Registro Oficial 449 jul 25, 2008. Disponible en: http://www21.ucsg.edu.ec:2337/Webtools/LexisFinder/DocumentVisualizer/DocumentVisualizer. aspx?id=PUBLICO-CONSTITUCION_DE_LA_REPUBLICA_DEL_ECUADOR\&query=constitucion $\% 20$ del\%20ecuador\#I_DXDataRow0

8. ISAGS. Sistemas de salud en Suramérica: desafíos para la universalidad la integralidad y la equidad. Rio de Janeiro: ISAGS; 2012.
9. Lopez-Cevallos DF, Chi C. Assessing the context of health care utilization in Ecuador: a spatial and multilevel analysis. BMC Health Serv Res. 12 de marzo de 2010;10:64.

10. Decreto Ejecutivo. N.1290 Ago, 2012.

11. Se crea agencia para la promoción y el control de la calidad, y la protección de usuarios y profesionales de la salud: ACESS - Ministerio de Salud Pública [Internet]. [citado 13 de diciembre de 2017]. Disponible en: http://www.salud.gob.ec/ se-crea-agencia-para-la-promocion-y-el-control-dela-calidad-y-la-proteccion-de-usuarios-y-profesionales-de-la-salud-acess/

12. ISAGS-UNASUR. Atención primaria de salud en Suramérica. Rio Jan ISAGS-UNASUR Retrieved Novemb. 2015;30:2015.

13. INEC. Anuario de Estadísticas Hospitalarias: Egresos y Camas 2014. 2014.

14. Ecuador [Internet]. Health in the Americas 2017. 2017 [citado 24 de diciembre de 2017]. Disponible en: http://www.paho.org/salud-en-las-americas2017/?post_t_es=ecuador

15. Organización Mundial de la Salud, Organización Panamericana de Salud. Monitoreo de la Política Andina de Planificación y Gestión de Recursos Humanos en Salud.

16. Medición y Monitoreo de Metas Regionales en Recursos Humanos en Salud | Observatorio Regional de Recursos Humanos de Salud [Internet]. [citado 30 de diciembre de 2017]. Disponible en: http:// www.observatoriorh.org/?q=node/368

17. Iturralde R, P.LA PPEDA, DONUM SF. Enfarma enferma y muere Problemas de la industria farmacéutica pública en el Ecuador. 2016. Disponible en: http://www.planv.com.ec/sites/default/files/ enfarma_enferma_y_muere.pdf.

18. Decreto Ejecutivo №. 181 http://www.ecuadorinmediato.com/Noticias/news_user_view/ jefe_de_estado_firma_decreto_para_creacion_de_ empresa_publica_de_farmacos--118686

19. La liquidación de un sueño llamado Enfarma EP | GK [Internet]. [citado 2 de enero de 2018]. Disponible en: https://gk.city/2016/06/20/la-liquidacionsueno-llamado-enfarma-ep/

20. Ortiz-Prado E, Ponce J, Cornejo-Leon F, StewartIbarra AM, Trujillo RH, Espín E, et al. Analysis of Health and Drug Access Associated with the Purchasing Power of the Ecuadorian Population. Glob J Health Sci. 19 de mayo de 2016;9(1):201.

21. World Bank. Health expenditure per capita (current US\$) | Data [Internet]. Data.worldbank.org. 2018. Disponible en: https://data.worldbank.org/ indicator/SH.XPD.PCAP?end=2014\&start $=1995 \&$ vie $\mathrm{w}=$ chart 
22. Out-of-pocket health expenditure (\% of total expenditure on health) | Data [Internet]. [citado 8 de noviembre de 2017]. Disponible en: https://data. worldbank.org/indicator/SH.XPD.OOPC.TO.ZS

23. Chang C. Evolución del Sistema de Salud de Ecuador. Buenas Practicas y Desafíos en su construcción en la última década. 2005-2014. Anales UNMSM, Vol. 78, Núm. 4 (2017), disponible en: http:// revistasinvestigacion.unmsm.edu.pe/index.php/ anales/article/view/14270

24. Cañizares R. El Sistema Nacional de Salud de Ecuador: Estudiarlo para mejorarlo. Alternativas. 15 de febrero de 2017;17(2):60-4.

25. "El 30 por ciento del presupuesto en salud se malgasta" [Internet]. [citado 24 de diciembre de 2017]. Disponible en: http://www.semana.com/ amp/entrevista-a-gerente-general-de-pfizer-paracolombia-y-venezuela/550668
26. López Tagle E, Santana Nazarit P. The 2010 earthquake in Chile: the response of the health system and international cooperation. Rev Panam Salud Pública. agosto de 2011;30(2):160-6.

27. Ministra Espinosa recibió aportes del Consejo Sectorial de Salud para construcción del Código de Salud - Ministerio de Salud Pública [Internet]. [citado 28 de diciembre de 2017]. Disponible en: http://www. salud.gob.ec/ministra-de-salud-recibio-aportesdel-consejo-sectorial-de-salud-para-construcciondel-codigo-de-salud/

28. Malo-Serrano M, Malo-Corral N. Health reform in Ecuador: never again the right to health as a privilege. Rev Peru Med Exp Salud Publica. diciembre de 2014;31(4):754-61. 